APS/PRL

\title{
Dome-shaped magnetic phase diagram of thermoelectric layered cobaltites
}

\author{
J. Sugiyama ${ }^{1}$ J. H. Brewer ${ }^{2}$, E. J. Ansaldo ${ }^{3}$, H. Itahara ${ }^{1}$, T. Tani ${ }^{1}$, \\ M. Mikami ${ }^{4} \dagger$ Y. Mori ${ }^{4}$, T. Sasaki ${ }^{4}$, S. Hébert, ${ }^{5}$, and A. Maignan ${ }^{5}$ \\ ${ }^{1}$ Toyota Central Research and Development Labs. Inc., Nagakute, Aichi 480-1192, Japan \\ ${ }^{2}$ TRIUMF, CIAR and Department of Physics and Astronomy, \\ University of British Columbia, Vancouver, BC, V6T $1 Z 1$ Canada \\ ${ }^{3}$ TRIUMF, 4004 Wesbrook Mall, Vancouver, BC, V6T $2 A 3$ Canada \\ ${ }^{4}$ Department of Electrical Engineering, Osaka University, Suita, Osaka 563-8577, Japan and \\ ${ }^{5}$ Laboratoire CRISMAT, CNRS/ISMRA/University of Caen, \\ 6 bd du Maréchal Juin, 14050 Caen Cedex, France
}

(Dated: June 5, 2018)

\begin{abstract}
Using muon spin spectroscopy we have found that, for both $\mathrm{Na}_{x} \mathrm{CoO}_{2}(0.6 \leq x \leq 0.9)$ and 3- and 4-layer cobaltites, a common low temperature magnetic state (which in some cases is manifest as an incommensurate spin density wave) forms in the $\mathrm{CoO}_{2}$ planes. Here we summarize those results and report a dome-shaped relation between the transition temperature into the low- $T$ magnetic state and the composition $x$ for $\mathrm{Na}_{x} \mathrm{CoO}_{2}$ and/or the high-temperature asymptotic limit of thermopower in the more complex 3- and 4-layer cobaltites. This behavior is explained using the Hubbard model on two-dimensional triangular lattice in the $\mathrm{CoO}_{2}$ plane.
\end{abstract}

PACS numbers: 76.75.+i, 75.30.Fv, 72.15.Jf, 75.30.Kz

Although the widespread current interest in the layered cobaltites [1, 2] was originally due mainly to their unique combination of high thermopower $S$ with metallic transport properties, 3, 4, 5, 6, 7, 8, 9, 10, 11, 12] which makes them one of the most promising systems for power applications, we have shown that they also display interesting and complex magnetic orderings, directly correlated with the enhanced thermopower. The richness of behavior of the layered cobaltites is due to their intrinsic structure, namely: electrically active triangular plane layers of $\mathrm{CoO}_{2}$, which are separated by a variety of intermediate structures; their relatively strong electronic correlations; and the fact that the structures between the $\mathrm{CoO}_{2}$ layers can be modified in a variety of ways to vary their dimensionality, ionic states, carrier doping in the $\mathrm{CoO}_{2}$ planes and the relevant interaction strengths.

In order to elucidate the magnetism in the $\mathrm{CoO}_{2}$ planes and the mechanism of the good thermoelectric properties, we have carried out positive muon spin rotation and relaxation $\left(\mu^{+} \mathrm{SR}\right)$ experiments on the layered cobaltites. As a result, we found the transition from a high-temperature paramagnetic to a lowtemperature commensurate or incommensurate spin density wave $\left(\mathrm{C}\right.$ - or IC-SDW) state for $\left[\mathrm{Ca}_{2} \mathrm{CoO}_{3}\right]_{0,6}^{\mathrm{RS}}\left[\mathrm{CoO}_{2}\right]$ below $\sim 100 \mathrm{~K},[13,14] \mathrm{Na}_{0.75} \mathrm{CoO}_{2}$ at $22 \mathrm{~K}$ 15] and $\left[\mathrm{Ca}_{2} \mathrm{Co}_{4 / 3} \mathrm{Cu}_{2 / 3} \mathrm{O}_{4}\right]_{0.62}^{\mathrm{RS}}\left[\mathrm{CoO}_{2}\right]$ below $\sim 200 \mathrm{~K},[16]$ where RS denotes the rocksalt-type subsystem. The common magnetic ordering is thus not always that of a classic frustrated AF (triangular planar arrangement of spins) system but rather a variety of states including disordered $\mathrm{AF}$ and (most common) an incommensurate spin density

\footnotetext{
*Electronic address: e0589@mosk.tytlabs.co.jp

${ }^{\dagger}$ Present address: National Institute of Advanced Industrial Science and technology, Ikeda, Osaka 563-8577, Japan.
}

wave (IC-SDW), $\mathrm{n}$ arising in parallel with other effects in the triangular lattice such as effective mass enhancement and the enhanced $S$ in the $\mathrm{Na}_{x} \mathrm{CoO}_{2}$ case. The more complex cobaltites exhibit, in addition, other magnetic orderings and even higher $S$.

Nevertheless, the transition temperature was scattered in the wide temperature range $(22-200 \mathrm{~K})$, and there was no clear relationship between $T_{\mathrm{SDW}}$ and structural properties and/or carrier concentration of the layered cobaltites. We back therefore to the basic system, $\mathrm{Na}_{x} \mathrm{CoO}_{2}$, to investigate the dependence of $T_{\text {SDW }}$ on $x$ by $\mu^{+} \mathrm{SR}$. Also, for the multilayer systems, we clarify the magnitude of $T_{\text {SDW }}$ as a function of the Co valence varied by the change in composition of the rocksalt-type subsystem. [8, 9, 10] For the $\mathrm{Na}_{x} \mathrm{CoO}_{2}$ case, we show that a model based on the Hubbard model for a triangular lattice of $\mathbf{S}=1 / 2$ Co ions with occupancy given by $x$ (correlated with band filling) 17,18$]$ is able to explain the dome shaped magnetic phase diagram shown below. Similar behavior is observed for the multilayer systems, where the increased two dimensionality due to the extra layers leads to higher $T_{\mathrm{SDW}}$ for the IC-SDW state and is correlated with higher $S$.

Single-crystal platelets of $\mathrm{Na}_{x} \mathrm{CoO}_{2}$ were prepared by a flux method. 19] Polycrystalline 3- and 4-layer cobaltites listed in Table 1 were synthesized by a solid state reaction technique 8, 9, 10, 13 or a reactive templated grain growth technique. 20] The $\mu^{+} \mathrm{SR}$ experiments were performed on the M15 and M20 surface muon beam lines at TRIUMF. The experimental setup is described in elsewhere. 21, 22]

Figure 1 shows zero-field (ZF-) $\mu^{+}$SR time spectra at $1.8 \mathrm{~K}$ for single crystal platelets of $\mathrm{Na}_{0.9} \mathrm{CoO}_{2}$. The top spectrum was obtained with the initial $\mu^{+}$spin direction $\overrightarrow{\boldsymbol{S}}_{\mu}(0)$ perpendicular to the $c$-axis and the bottom one with $\overrightarrow{\boldsymbol{S}}_{\mu}(0)$ parallel to $c$. A clear oscillation due to quasi- 


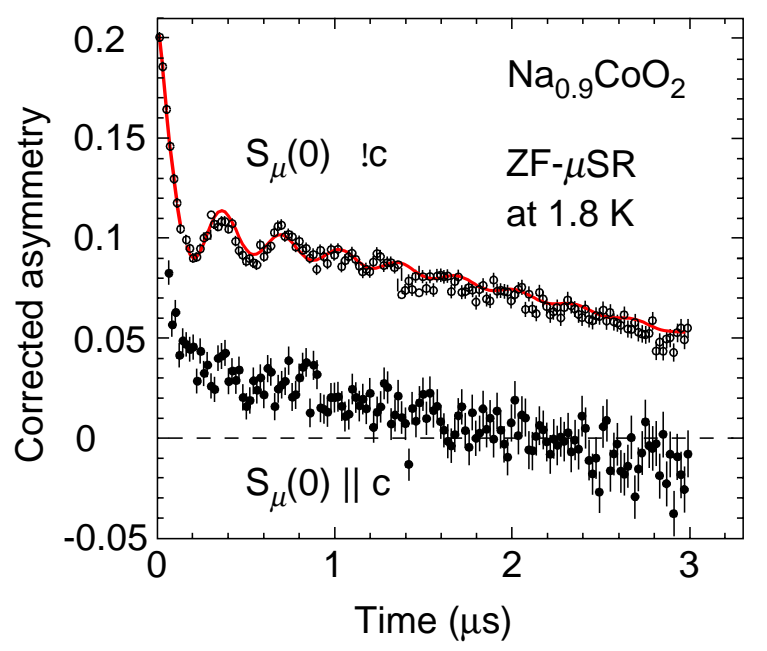

FIG. 1: $\mathrm{ZF}-\mu^{+} \mathrm{SR}$ time spectra of single crystal platelets of $\mathrm{Na}_{0.9} \mathrm{CoO}_{2}$ at $1.8 \mathrm{~K}$. The configurations of the sample and the initial muon spin direction $\overrightarrow{\boldsymbol{S}}_{\mu}(0)$ are (top) $\overrightarrow{\boldsymbol{S}}_{\mu}(0) \perp \hat{\boldsymbol{c}}$ and (bottom) $\overrightarrow{\boldsymbol{S}}_{\mu}(0) \| \hat{\boldsymbol{c}}$. The bottom spectrum is offset below to be seen clearly.

static internal fields is observed only for $\overrightarrow{\boldsymbol{S}}_{\mu}(0)$ perpendicular to $\boldsymbol{c}$. The muon signal is fitted best by a zerothorder Bessel function of the first kind, $J_{0}\left(\omega_{\mu} t\right)$, which describes the evolution of the muon polarization in an IC-SDW field distribution; 21, 22 we therefore conclude that $\mathrm{Na}_{0.9} \mathrm{CoO}_{2}$ undergoes a magnetic transition from a paramagnetic state to an IC-SDW state at $T_{\mathrm{SDW}}=19 \mathrm{~K}$. The absence of a clear oscillation for the $\overrightarrow{\boldsymbol{S}}_{\mu}(0) \| \boldsymbol{c}$ case indicates that the internal field $\boldsymbol{H}_{\text {int }}$ is roughly parallel to the $c$-axis. Because of the strong anisotropy, the ICSDW is most likely to propagate in the $c$ plane (i.e. in the $\mathrm{CoO}_{2}$ plane), with oscillating moments directed along the $c$-axis.

Figure 2 shows the temperature dependence of the muon precession frequency $\nu_{\mu}=\omega_{\mu} / 2 \pi$ for the single crystal platelets of $\mathrm{Na}_{0.9} \mathrm{CoO}_{2}$. Here $\nu_{\mu}$ is the order parameter of the transition and its $T$ dependence is well described by the BCS weak coupling expression for such order parameters, as expected for the IC-SDW state. 23. The magnetic phase diagram (Fig. 3) of $\mathrm{Na}_{x} \mathrm{CoO}_{2}$ can thus be sketched from the $\mu^{+} \mathrm{SR}$ results for polycrystalline samples with $x=0.65$ and $0.75[15]$ and the present $x=0.6$ and 0.9 crystals. Recent compositional and chemical titration analyses indicated that the oxygen deficiency $\delta$ in $\mathrm{Na}_{x} \mathrm{CoO}_{2-\delta}$ is negligibly small even for the $x=0.9$ sample. 19] Hence, the average Co valence can be directly calculated from $x$. As $x$ increases from 0.6 (i.e. the Co valence decreases from 3.4), the magnitude of $T_{\mathrm{SDW}}$ increases up to around $x=0.8$, then decreases with further increasing $x$. As a result, we obtain the dome-shaped relationship of Figure 3 , between $T_{\mathrm{SDW}}$ and $x$, viz. the Co valence for $\mathrm{Na}_{x} \mathrm{CoO}_{2}$.

The $x=1$ end member of $\mathrm{Na}_{x} \mathrm{CoO}_{2}$, that is, a fully

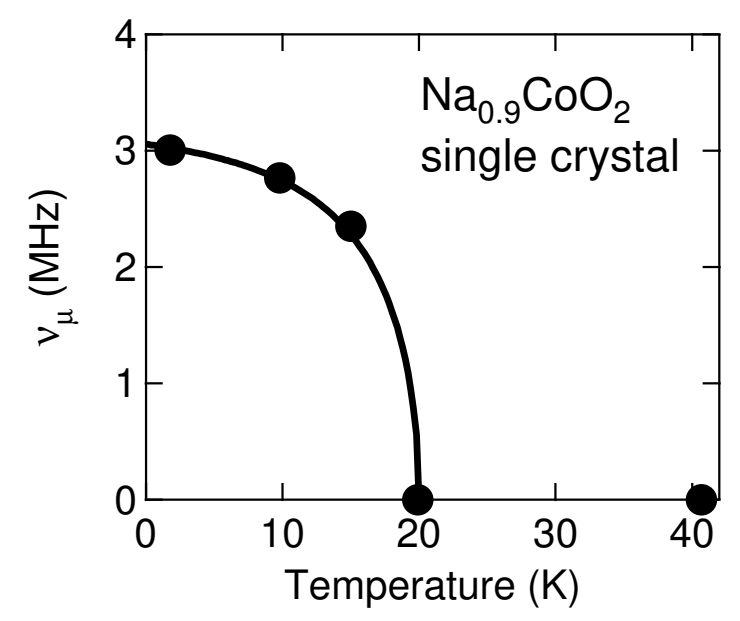

FIG. 2: Temperature dependence of the muon precession frequency $\nu_{\mu}$ in $\mathrm{Na}_{0.9} \mathrm{CoO}_{2}$. The solid line represents the temperature dependence of the BCS gap energy.

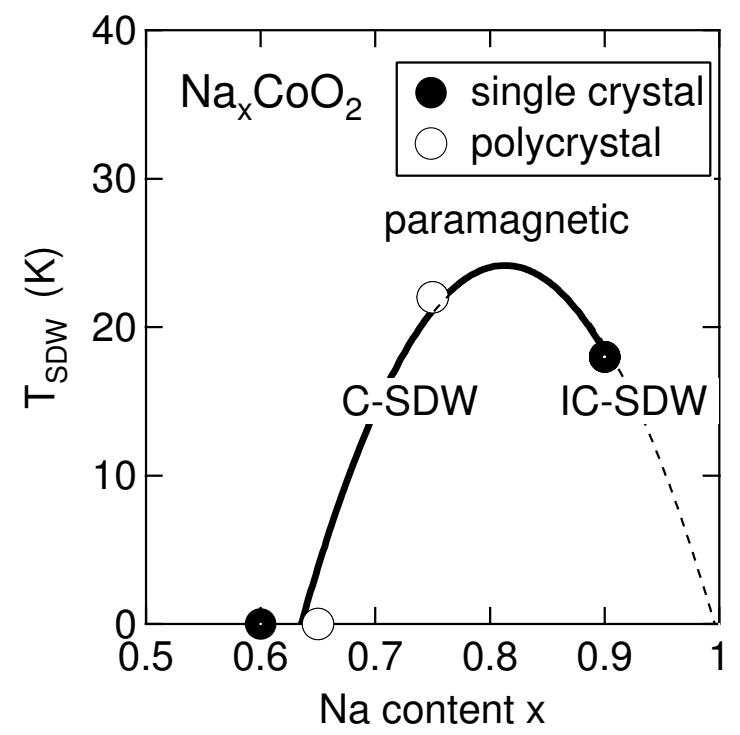

FIG. 3: Phase diagram of $\mathrm{Na}_{x} \mathrm{CoO}_{2}$ determined by the $\mu^{+} \mathrm{SR}$ experiments. Solid and open circles represent the present results on single crystals and polycrystalline samples, respectively. The point at $x=1$ is extrapolated from the data on the related compound $\left.\mathrm{LiCoO}_{2} \cdot 24\right]$

occupied $\mathrm{NaCoO}_{2}$ phase, cannot be prepared by conventional solid state reaction and/or flux techniques, whereas the related compound $\mathrm{LiCoO}_{2}$ is easily obtained. The structure of $\mathrm{LiCoO}_{2}$ is isomorphous with $\alpha-\mathrm{NaFeO}_{2}$ $(R 3 m)$, almost the same as that of $\mathrm{Na}_{x} \mathrm{CoO}_{2}$, and it is reported to be diamagnetic down to $4.2 \mathrm{~K}$. 24] This behavior is expected, because the $\mathrm{Co}^{3+}$ ions in $\mathrm{LiCoO}_{2}$ are in a low-spin state $t_{2 g}^{6}$, as for $\mathrm{Na}_{x} \mathrm{CoO}_{2}$. Therefore, $\mathrm{Na}_{x} \mathrm{CoO}_{2}$ is also expected to lack magnetically ordered states. 
The occupancy of $\mathrm{Co}^{4+}$ spins $(\mathbf{S}=1 / 2)$ in the twodimensional triangular lattice (2DTL) increases with decreasing $x$. Thus the other end member, $\mathrm{Na}_{0} \mathrm{CoO}_{2}$, would be a half filled 2DTL. In other words, every lattice site is occupied by an $\mathbf{S}=1 / 2$ spin. The Hubbard model within a mean field approximation can be used for explaining the magnetism of such a system, with the Hamiltonian $\mathcal{H}$ given by [17, 18]

$$
\mathcal{H}=-t \sum_{<i j>\sigma} c_{i \sigma}^{\dagger} c_{j \sigma}+U \sum_{i} n_{i \uparrow} n_{i \downarrow}
$$

where $c_{i \sigma}^{\dagger}\left(c_{j \sigma}\right)$ creates (destroys) an electron with spin $\sigma$ on site $i, n_{i \sigma}=c_{i \sigma}^{\dagger} c_{i \sigma}$ is the number operator, $t$ is the nearest-neighbor hopping amplitude and $U$ is the Hubbard on-site repulsion. The electron filling $n$ is defined as $n=(1 / 2 N) \sum_{i}^{N} n_{i}$, where $N$ is the total number of sites.

At $T=0$ and $n=0.5$ (i.e., $\mathrm{Na}_{0} \mathrm{CoO}_{2}$ ), as $U$ increases from 0 , the system is a paramagnetic metal up to $U / t=3.97$ due to geometrical frustration, then changes into a metal with a spiral IC-SDW, and then at $U / t=5.27$ a first-order metal-insulator transition occurs. 17] The lack of magnetic transitions for $\mathrm{Na}_{x} \mathrm{CoO}_{2}$ with $x=0.6$ and 0.65 suggests that $U / t \leq 3.97$. This means that $\mathrm{Na}_{x} \mathrm{CoO}_{2}$ is unlikely to be a typical strongly correlated electron system, because $U \gg t$ for such a system. The calculations 18] also predict that, as $n$ increases from 0 , the magnitude of $U / t$ at the boundary between the paramagnetic and SDW phases decreases, with increasing slope $(d(U / t) / d n)$ up to $n=0.75$. Even for $U / t=0$, the SDW phase is stable at $n=0.75$. The value of $U / t$ then increases with further increasing $n$, with decreasing slope. Therefore, the dome-shaped phase diagram of Fig. 3 is qualitatively explained by the calculations, although the measured maximum of the dome is located around $x=0.8$ (i.e. $n=0.9$ ). This is likely due to the simple band structure assumed in the above calculation, while calculations for $\mathrm{Na}_{x} \mathrm{CoO}_{2}$ suggest more complicated band structure. 25.

For the related compounds $\left[\mathrm{Ca}_{2} \mathrm{CoO}_{3}\right]_{0.62}^{\mathrm{RS}}\left[\mathrm{CoO}_{2}\right]$ and $\left[\mathrm{Ca}_{2} \mathrm{Co}_{4 / 3} \mathrm{Cu}_{2 / 3} \mathrm{O}_{4}\right]_{0.62}^{\mathrm{RS}}\left[\mathrm{CoO}_{2}\right]$, (i.e. 3-layer and 4-layer cobaltites, where RS denotes the rocksalt-type susbsystem), the IC-SDW transition was also observed as a common behavior in the $\mathrm{CoO}_{2}$ planes. 13, 14, 16] We have studied by $\mu^{+}$SR the dependence of the IC-SDW transition on the Co valence for the variety of layered cobaltites shown in Table 1. It should be noted that the magnetic susceptibility $\chi(T)$ curves for the 3 - and 4-layer samples lacked a marked change at either the $T_{\mathrm{SDW}}^{\mathrm{on}}$ or $T_{\mathrm{SDW}}^{\mathrm{end}}$ detected by $\mu^{+}$SR.

There are two Co sites in these layered cobaltite lattices, one in the rocksalt-type subsystem and the other in the $\mathrm{CoO}_{2}$ plane. Therefore it is difficult to determine the Co valence in the $\mathrm{CoO}_{2}$ plane by $\chi$ measurements or chemical titration techniques. Since the Hall coefficients $R_{\mathrm{H}}$ of these cobaltites are reported to depend strongly on temperature, 11, 19] it is also difficult to estimate the carrier concentration in the $\mathrm{CoO}_{2}$ plane. On the other hand,

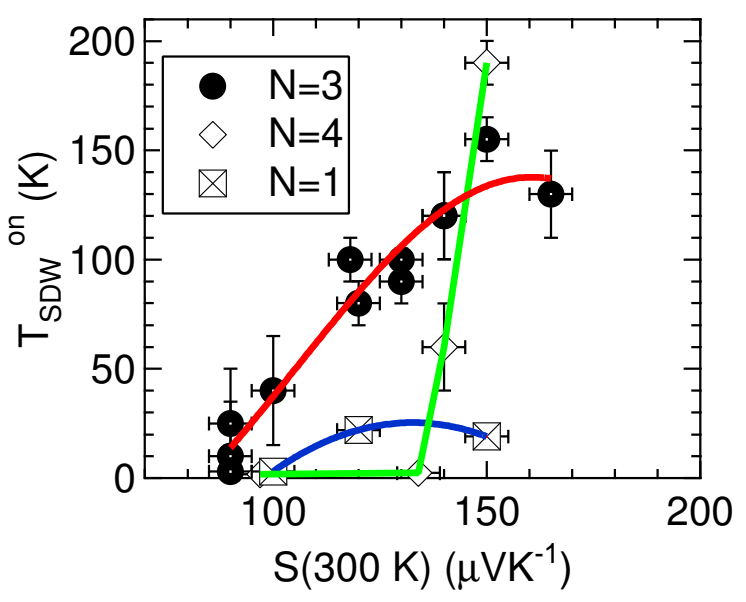

FIG. 4: The relationship between $T_{\mathrm{SDW}}^{\mathrm{on}}$ and thermopower $S$ at $300 \mathrm{~K}$. Solid circles represent the data for the cobaltites with a triple rocksalt-type subsystem, open diamonds a quadruple rocksalt-type subsystem and crossed squares $\mathrm{Na}_{x} \mathrm{CoO}_{2}$.

the magnitude of $S$ in the layered cobaltites is almost independent of $T$ above $200 \mathrm{~K}$. [7, 8, 9, 10, 11, 12] Therefore, we use the value of $S$ at $300 \mathrm{~K}$ as an indicator of the Co valence in the $\mathrm{CoO}_{2}$ plane for these cobaltites. The modified Heikes formula gives $S$ at the high-temperature $\operatorname{limit}\left(S_{T \rightarrow \infty}\right)$ as $[26]$

$$
S_{T \rightarrow \infty}=-\frac{k_{\mathrm{B}}}{e} \ln \left(\frac{g_{3}}{g_{4}} \frac{y}{1-y}\right)
$$

where $k_{\mathrm{B}}$ is the Boltzmann constant, $e$ is the elementary charge and $g_{3}$ and $g_{4}$ are the numbers of the spin configurations of $\mathrm{Co}^{3+}$ and $\mathrm{Co}^{4+}$ ions, respectively, and $y$ is the ratio $\mathrm{Co}^{4+} /\left(\mathrm{Co}^{3+}+\mathrm{Co}^{4+}\right)$. Since both $\mathrm{Co}^{3+}$ and $\mathrm{Co}^{4+}$ are in the low-spin state $\left(t_{2 g}^{6}\right.$ and $\left.t_{2 g}^{5}\right)$ and $g_{3}=1$ and $g_{4}=6$, we can convert $S(300 \mathrm{~K})$ to $y$, assuming that $S(300 \mathrm{~K})=S_{T \rightarrow \infty}$.

Figure 4 shows $T_{\mathrm{SDW}}^{\mathrm{on}}$ as a function of $S(300 \mathrm{~K})$ for the all cobaltites listed in Table 1. A clear dome-shaped relation is thus observed for all the available cobaltites with a variable number of layers $(N)$ between the two adjacent $\mathrm{CoO}_{2}$ planes. As $N$ increases from 1 , the $T_{\mathrm{SDW}}^{\mathrm{on}}-v s .-S(300 \mathrm{~K})$ curve shifts towards higher temperature. This is due to the increased two-dimensionality induced by the increase in the interlayer distance between $\mathrm{CoO}_{2}$ planes. Also, the large observed transition widths $(50-130 \mathrm{~K})$ are consistent with enhanced two-dimensionality and resulting spin fluctuations. Phenomenologically, the phase diagram is very similar to the well-known relationship between the superconducting $T_{\mathrm{c}}$ and the $\mathrm{Cu}$ valence in the high- $T_{\mathrm{c}}$ cuprates. Actually, both SDW and superconducting transitions are induced by an intrinsic instability of an electron system; in other words, as $T$ decreases, an energy gap appears at $T_{\text {SDW }}$ and/or $T_{\mathrm{c}}$ to minimize the internal energy for both cases. 
TABLE I: Parameters of the magnetic transition and thermopower $S$ of several cobaltites; the number of the layers between the two adjacent $\left[\mathrm{CoO}_{2}\right]$ planes $(N)$, the onset and endpoint temperature of the magnetic transition detected by the $\mu^{+}$SR experiments and $S$ at $300 \mathrm{~K}$. nd $\geq 3.3$ means not detected down to $\left.3.3 \mathrm{~K} .{ }^{*}{ }^{* *}\right)$ indicates the sample showing a clear muon precession due to the IC-SDW (C-SDW) field in a ZF- $\mu^{+}$SR spectrum.

\begin{tabular}{|c|c|c|c|c|}
\hline cobaltite & $N$ & $T_{\mathrm{SDW}}^{\mathrm{on}}(\mathrm{K})$ & $T_{\mathrm{SDW}}^{\mathrm{end}}(\mathrm{K})$ & $S(300 \mathrm{~K})\left(\mu \mathrm{VK}^{-1}\right)$ \\
\hline$\left[\mathrm{Ca}_{2} \mathrm{CoO}_{3}\right]_{0.62}^{\mathrm{RS}}\left[\mathrm{CoO}_{2}\right]$ & 3 & $100 \pm 5$ & $30 \pm 5^{*}$ & 130 \\
\hline$\left[\mathrm{Ca}_{1.8} \mathrm{Bi}_{0.2} \mathrm{CoO}_{3}\right]_{x}^{\mathrm{RS}}\left[\mathrm{CoO}_{2}\right]$ & 3 & $120 \pm 20$ & $70 \pm 10^{*}$ & 140 \\
\hline$\left[\mathrm{Ca}_{1.8} \mathrm{Y}_{0.2} \mathrm{CoO}_{3}\right]_{x}^{\mathrm{RS}}\left[\mathrm{CoO}_{2}\right]$ & 3 & $120 \pm 20$ & $70 \pm 10^{*}$ & 140 \\
\hline$\left[\mathrm{Ca}_{1.8} \mathrm{Sr}_{0.2} \mathrm{CoO}_{3}\right]_{x}^{\mathrm{RS}}\left[\mathrm{CoO}_{2}\right]$ & 3 & $100 \pm 10$ & $45 \pm 10^{*}$ & 118 \\
\hline$\left[\mathrm{Pb}_{0.4} \mathrm{Co}_{0.6} \mathrm{Ca}_{2} \mathrm{O}_{3}\right]_{0.62}^{\mathrm{RS}}\left[\mathrm{CoO}_{2}\right]$ & 3 & $130 \pm 20$ & $40 \pm 10^{*}$ & 165 \\
\hline$\left[\mathrm{Pb}_{0.7} \mathrm{Co}_{0.4} \mathrm{Sr}_{1} \mathrm{Ca}_{0.9} \mathrm{O}_{3}\right]_{0.58}^{\mathrm{RS}}\left[\mathrm{CoO}_{2}\right]$ & 3 & $90 \pm 10$ & $10 \pm 10$ & 130 \\
\hline$\left[\mathrm{Tl}_{1.01} \mathrm{Co}_{0.11} \mathrm{Sr}_{1.88} \mathrm{O}_{3}\right]_{0.56}^{\mathrm{RS}}\left[\mathrm{CoO}_{2}\right]$ & 3 & $25 \pm 5$ & $\mathrm{nd} \geq 1.7$ & 90 \\
\hline$\left[\mathrm{Tl}_{1.18} \mathrm{Co}_{0.03} \mathrm{Sr}_{1.78} \mathrm{O}_{3}\right]_{0.57}^{\mathrm{RS}}\left[\mathrm{CoO}_{2}\right]$ & 3 & $\mathrm{nd} \geq 2.5$ & $\mathrm{nd} \geq 2.5$ & 90 \\
\hline$\left[\mathrm{Hg}_{0.4} \mathrm{Co}_{0.6} \mathrm{Sr}_{2} \mathrm{O}_{3}\right]_{0.56}^{\mathrm{RS}}\left[\mathrm{CoO}_{2}\right]$ & 3 & $40 \pm 25$ & $5 \pm 5$ & 100 \\
\hline$\left[\mathrm{Ti}_{0.4} \mathrm{Co}_{0.6} \mathrm{Ca}_{2} \mathrm{O}_{3}\right]_{0.62}^{\mathrm{RS}}\left[\mathrm{CoO}_{2}\right]$ & 3 & $150 \pm 10$ & $15 \pm 5$ & 150 \\
\hline$\left[\mathrm{Ca}_{2} \mathrm{Co}_{4 / 3} \mathrm{Cu}_{2 / 3} \mathrm{O}_{4}\right]_{0.62}^{\mathrm{RS}}\left[\mathrm{CoO}_{2}\right]$ & 4 & $190 \pm 10$ & $145 \pm 10^{*}$ & 150 \\
\hline$\left[\mathrm{Ca}_{2} \mathrm{Bi}_{1.7} \mathrm{Co}_{0.3} \mathrm{O}_{4}\right]_{0.60}^{\mathrm{RS}}\left[\mathrm{CoO}_{2}\right]$ & 4 & $60 \pm 20$ & $\mathrm{nd} \geq 5$ & 140 \\
\hline $\mathrm{Na}_{0.75} \mathrm{CoO}_{2}$ & 1 & $22 \pm 0.5$ & $22 \pm 1^{* *}$ & 120 \\
\hline $\mathrm{Na}_{0.65} \mathrm{CoO}_{2}$ & 1 & $\mathrm{nd} \geq 2.5$ & $\mathrm{nd} \geq 2.5$ & 100 \\
\hline $\mathrm{Na}_{0.6} \mathrm{CoO}_{2}$ & 1 & $\mathrm{nd} \geq 4.3$ & $\mathrm{nd} \geq 4.3$ & 100 \\
\hline
\end{tabular}

Therefore, it is reasonable to expect a similar relationship between transition temperature and carrier concentration for both the magnetic cobaltites and the superconducting cuprates.

Furthermore, the average Co valence for the maximum $T_{\text {SDW }}$ indicates the optimal filling to induce an SDW transition at high temperatures and enhance the effective mass of charge carriers through the AF interaction between spins. In other words, this dome relation provides important guidance in the search for improved thermoelectric properties of the layered cobaltites.

We thank S.R. Kreitzman, B. Hitti, D.J. Arseneau, Y. Seno, K. Dohmae, C. Xia, H. Nozaki, H. Hazama, J.
Chakhalian, D. Liu, A. I.-Najafabadi and S. D. LaRoy for help with the $\mu^{+} \mathrm{SR}$ experiments. Also, we appreciate R. Asahi, U. Mizutani, H. Ikuta, T. Takeuchi and K. Machida for discussions. This work was supported at Toyota CRDL by joint research and development with International Center for Environmental Technology Transfer in 2002-2004, commissioned by the Ministry of Economy Trade and Industry of Japan, at UBC by the Canadian Institute for Advanced Research, the Natural Sciences and Engineering Research Council of Canada, and at TRIUMF by the National Research Council of Canada.
[1] K. Takada et al. Nature 422, 53 (2003).

[2] R. E. Schaak et al., Nature 424, 527 (2003).

[3] J. Molenda et al., Solid State Ionics 12, 473 (1989).

[4] H. Yakabe et al., in Proc. 16th Int. Conf. Thermoelectrics, (IEEE, Piscataway, 1998) pp. 523-527.

[5] I. Terasaki, Y. Sasago, and K. Uchinokura, Phys. Rev. B 56, R12685 (1997)

[6] R. Funahashi et al., Jpn. J. Appl. Phys. 39, L1127 (2000).

[7] A. C. Masset et al. Phys. Rev. B 62, 166 (2000).

[8] S. Hébert et al., Phys. Rev. B 64, 172101 (2001).

[9] A. Maignan et al., J. Appl. Phys. 92, 1964 (2002).

[10] D. Pelloquin et al., J. Solid State Chem. 170, 374 (2003).

[11] T. Yamamoto, K. Uchinokura, and I. Tsukada, Phys. Rev. B 65, 184434 (2002).

[12] Y. Miyazaki et al., Jpn. J. Appl. Phys. 41, L849 (2002).
[13] J. Sugiyama et al., Phys. Rev. B 66, 134413 (2002).

[14] J. Sugiyama et al., Phys. Rev. B 68, 134423 (2003).

[15] J. Sugiyama et al., Phys. Rev. B 67, 214420 (2003).

[16] J. Sugiyama et al., (2003) unpublished.

[17] H. R. Krishnamurthy et al., Phys. Rev. Lett. 64, 950 (1990).

[18] M. Fujita, M. Ichimura and K. Nakao, J. Phys. Soc. Jpn. 60, 2831 (1991); M. Fujita, T. Nakanishi, and K. Machida, Phys. Rev. B 45, 2190 (1992).

[19] M. Mikami, thesis, Osaka University (2003) in Japanese.

[20] T. Tani et al., J. Mater. Chem. 13, 1865 (2003).

[21] Y. J. Uemura, Muon Science edited by S. L. Lee et al., (Institute of Physics Publishing, Bristol, 1999) pp. 85114.

[22] G. M. Kalvius, D. R. Noakes, and O. Hartmann, Hand- 
book on the Physics and Chemistry of Rare Earths edited by K. A. Gschneidner Jr., L. Eyring, and G. H. Lander, (North-Holland, Amsterdam, 2001) vol. 32, chap. 206.

[23] G. Grüner, Density Waves in Solids (Addison-WesleyLongmans, Reading, 1994) chap. 4.

[24] I. Tomeno, and M. Oguchi, J. Phys. Soc. Jpn. 67, 318
(1998).

[25] D. J. Singh, Phys. Rev. B 61, 13397 (2000).

[26] W. Koshibae, K. Tsutsui, and S. Maekawa, Phys. Rev. B 62, 6869 (2000). 